\title{
On the Location of Zeros of Analytic Functions
}

\author{
M. H. Gulzar \\ P. G. Department of Mathematics University of Kashmir, Srinagar 190006
}

Abstract: In this paper we consider a certain class of analytic functions whose coefficients are restricted to certain conditions, and find some interesting zero-free regions for them. Our results generalise a number of already known results in this direction.

Mathematics Subject Classification: 30C10,30C15

Key-words and phrases: Analytic Function, Coefficients,Zeros

\section{INTRODUCTION AND STATEMENT OF RESULTS}

Regarding the zeros of analytic functions, Aziz and Shah [2]proved the following results:

Theorem A: Let $f(z)=\sum_{j=0}^{\infty} a_{j} z^{j} \neq 0$ be a function analytic for $|z| \leq t$ and for some $k \geq 1$,

$$
k a_{0} \geq t a_{1} \geq t^{2} a_{2} \geq \ldots . . .
$$

Then $\mathrm{f}(\mathrm{z})$ does not vanjsh in

$$
\left|z-\left(\frac{k-1}{2 k-1}\right) t\right| \leq \frac{k t}{2 k-1} \text {. }
$$

Theorem B: Let $f(z)=\sum_{j=0}^{\infty} a_{j} z^{j} \neq 0$ be a function analytic for $|z| \leq t$ and

$$
\mid\left(a_{1}-t a_{2}\right)+\left(a_{2}-t a_{3}\right) z+\left(a_{3}-t a_{4}\right) z^{2}+\ldots . . \leq \frac{M}{t} \text { for }|z|=t .
$$

Then $\mathrm{f}(\mathrm{z})$ does not vanish in $|z|<R$, where

$$
R=\frac{1}{2 M}\left\{-\left|a_{0}-t a_{1}\right|+\sqrt{\left|a_{0}-t a_{1}\right|^{2}-4\left|a_{0}\right| M}\right\} .
$$

In this paper we are going to give generalizations of the above mentioned results. More precisely, we shall prove the following results:

Theorem 1: Let $f(z)=\sum_{j=0}^{\infty} a_{j} z^{j} \neq 0$ be a function analytic for $|z| \leq t$ and for some $\rho \geq 0$,

$$
\rho+a_{0} \geq t a_{1} \geq t^{2} a_{2} \geq \ldots \ldots .
$$

Then $\mathrm{f}(\mathrm{z})$ does not vanish in

$$
\left|z-\left(\frac{\rho}{2 \rho+a_{0}}\right) t\right|<\left(\frac{\rho+a_{0}}{2 \rho+a_{0}}\right) t .
$$

Remark 1: Taking $\rho=(k-1) a_{0}$, Theorem 1 reduces to Theorem A.

Taking $\rho=0$, we get the following result proved earlier by Aziz and Mohammad [1] :

Corollary 1: : Let $f(z)=\sum_{j=0}^{\infty} a_{j} z^{j} \neq 0$ be a function analytic for $|z| \leq t$ and

$$
0<a_{0} \geq t a_{1} \geq t^{2} a_{2} \geq \ldots \ldots .
$$

Then $\mathrm{f}(\mathrm{z})$ does not vanish in $|z|<t$. 
Theorem 2: Let $f(z)=\sum_{j=0}^{\infty} a_{j} z^{j} \neq 0$ be a function analytic for $|z| \leq t$ with $a_{j}=\alpha_{j} e^{i \phi}+\beta_{j} e^{i \psi}, \mathrm{j}=0,1,2, \ldots \ldots, \mathrm{n}$, and

$$
\begin{aligned}
& \mid\left(\alpha_{1}-t \alpha_{2}\right)+\left(\alpha_{2}-t \alpha_{3}\right) z+\left(\alpha_{3}-t \alpha_{4}\right) z^{2}+\ldots . . \leq \frac{M_{1}}{t} \text { for }|z|=t, \\
& \mid\left(\beta_{1}-t \beta_{2}\right)+\left(\beta_{2}-t \beta_{3}\right) z+\left(\beta_{3}-t \beta_{4}\right) z^{2}+\ldots . . \leq \frac{M_{2}}{t} \text { for }|z|=t .
\end{aligned}
$$

Then $\mathrm{f}(\mathrm{z})$ does not vanish in $|z|<R$, where

$$
R=\frac{1}{2\left(M_{1}+M_{2}\right)}\left\{-\left|a_{0}-t a_{1}\right|+\sqrt{\left|a_{0}-t a_{1}\right|^{2}+4\left|a_{0}\right|\left(M_{1}+M_{2}\right)}\right\} t .
$$

Remark 2: If $\beta_{j}=0, \forall j=0,1, \ldots ., n$, so that $M_{2}=0$, Theorem 2 reduces to Theorem $\mathrm{B}$ by taking $M_{1}=M$.

The following results are immediate consequences of Theorem 2 :

Corollary 2: : Let $f(z)=\sum_{j=0}^{\infty} a_{j} z^{j} \neq 0$ be analytic for $|z| \leq t$ with $a_{j}=\alpha_{j} e^{i \phi}+\beta_{j} e^{i \psi}, \mathrm{j}=0,1,2, \ldots \ldots, \mathrm{n}$, and for some $k \geq 1$

$$
t \alpha_{1} \leq t^{2} \alpha_{2} \leq \ldots \ldots . \leq t^{k} \alpha_{k} \geq t^{k+1} \alpha_{k+1} \geq \ldots \ldots . .
$$

Then $\mathrm{f}(\mathrm{z})$ does not vanish in

$$
|z|<\frac{1}{2 M_{k}}\left\{-\left|a_{0}-t a_{1}\right|+\sqrt{\left.\left|a_{0}-t a_{1}\right|^{2}+4\left|a_{0}\right| M_{k}\right)}\right\} t
$$

where

$$
M_{k}=2 t^{k} \alpha_{k}-t \alpha_{1}+t\left|\beta_{1}\right|+2 \sum_{j=2}^{\infty}\left|\beta_{j}\right| t^{j}
$$

Corollary 3: : Let $f(z)=\sum_{j=0}^{\infty} a_{j} z^{j} \neq 0$ be analytic for $|z| \leq t$ with $a_{j}=\alpha_{j} e^{i \phi}+\beta_{j} e^{i \psi}, \mathrm{j}=0,1,2, \ldots \ldots, \mathrm{n}$, and for some $k \geq 1$

$$
\begin{aligned}
& t \alpha_{1} \leq t^{2} \alpha_{2} \leq \ldots \ldots . \leq t^{k} \alpha_{k} \geq t^{k+1} \alpha_{k+1} \geq \ldots \ldots . . \\
& t \beta_{1} \leq t^{2} \beta_{2} \leq \ldots \ldots \leq t^{k} \beta_{k} \geq t^{k+1} \beta_{k+1} \geq \ldots \ldots . .
\end{aligned}
$$

Then $\mathrm{f}(\mathrm{z})$ does not vanish in

$$
|z|<\frac{1}{2 M_{k}}\left\{-\left|a_{0}-t a_{1}\right|+\sqrt{\left.\left|a_{0}-t a_{1}\right|^{2}+4\left|a_{0}\right| M_{k}\right)}\right\} t
$$

where

$$
M_{k}=2 t^{k} \alpha_{k}-t \alpha_{1}+2 t^{k} \beta_{k}-t \beta_{1} .
$$

Taking k=1in Cor.2, and noting that $M_{1}=t a_{1}$ and $M_{2}=t a_{2}$, we get the following result from Cor.2:

Corollary 4 : Let $f(z)=\sum_{j=0}^{\infty} a_{j} z^{j} \neq 0$ be analytic for $|z| \leq t$ with $a_{j}=\alpha_{j} e^{i \phi}+\beta_{j} e^{i \psi}, \mathrm{j}=0,1,2, \ldots \ldots, \mathrm{n}$, and 


$$
\begin{aligned}
& 0<t \alpha_{1} \geq t^{2} \alpha_{2} \geq \ldots . . . \\
& 0<t \beta_{1} \geq t^{2} \beta_{2} \geq \ldots . .
\end{aligned}
$$

Then $\mathrm{f}(\mathrm{z})$ does not vanish in

$$
|z|<\frac{1}{2\left(\alpha_{1}+\beta_{1}\right)}\left\{-\left|a_{0}-t a_{1}\right|+\sqrt{\left.\left|a_{0}-t a_{1}\right|^{2}-4\left|a_{0}\right|\left(\alpha_{1}+\beta_{1}\right) t\right)}\right\} .
$$

Taking k=1and $\beta_{j}=0, \forall j=0,1, \ldots ., n$,in Cor.2, and noting that $M_{1}=t a_{1}$, Cor.3 reduces to Cor.1.

\section{PROOFS OF THEOREMS}

Proof of Theorem 1: Since $f(z)=\sum_{j=0}^{\infty} a_{j} z^{j} \neq 0$ is analytic for $|z| \leq t$, therefore $\lim _{j \rightarrow \infty} a_{j} t^{j}=0$. Consider the function

$$
\begin{aligned}
F(z) & =(z-t) f(t z) \\
& =(z-t)\left(a_{0}+a_{1} t z+a_{2} t^{2} z^{2}+\ldots \ldots\right) \\
& =-t a_{0}+\left(a_{0}-a_{1} t\right) z+\left(a_{1} t-a_{2} t^{2}\right) z^{2}+\ldots \ldots \\
& =-t a_{0}-\rho z+\left(\rho+a_{0}-a_{1} t\right) z+\left(a_{1} t-a_{2} t^{2}\right) z^{2}+\ldots \ldots . \\
& =-t a_{0}-\rho z+G(z),
\end{aligned}
$$

where

$$
G(z)=\left(\rho+a_{0}-a_{1} t\right) z+z \sum_{j=2}^{\infty}\left(a_{j-1}-a_{j} t\right) z^{j-1} .
$$

For $|z|=t$,

$$
\begin{aligned}
& |G(z)| \leq\left(\rho+a_{0}-a_{1} t\right) t+t\left[\left(a_{1}-a_{2} t\right) t+\left(a_{2}-a_{3} t\right) t^{2}+\ldots \ldots .\right] \\
& \quad=\left(\rho+a_{0}\right) t .
\end{aligned}
$$

Since $\mathrm{f}(\mathrm{z})$ is analytic for $|z| \leq t, \mathrm{G}(\mathrm{z})$ is analytic for $|z| \leq t$ and $\mathrm{G}(0)=0$, we apply Schwarz lemma to $\mathrm{G}(\mathrm{z})$ to get

$$
|G(z)| \leq\left(\rho+a_{0}\right)|z| \quad \text { for }|z| \leq t .
$$

Hence it follows that

$$
\begin{aligned}
& |F(z)| \geq\left|t a_{0}+\rho z\right|-|G(z)| \\
& \quad \geq\left|a_{0}\right|\left\{t+\frac{\rho z}{a_{0}}\left|-\left(\frac{\rho+a_{0}}{\left|a_{0}\right|}\right)\right| z \mid\right\} \\
& \quad>0
\end{aligned}
$$

if

$$
\left(\frac{\rho+a_{0}}{\left|a_{0}\right|}\right)|z|<\left|t+\frac{\rho z}{a_{0}}\right| .
$$

It is easy to see that the region defined by $\left(\frac{\rho+a_{0}}{\left|a_{0}\right|}\right)|z|<\left|t+\frac{\rho z}{a_{0}}\right|$ is precisely the disk

$$
\left\{z ;\left|z-\left(\frac{\rho}{2 \rho+a_{0}}\right) t\right|<\left(\frac{\rho+a_{0}}{2 \rho+a_{0}}\right) t\right\} .
$$

Hence it follows that $\mathrm{F}(\mathrm{z})$ and therefore $\mathrm{f}(\mathrm{z})$ does not vanish in the disk

$$
\left|z-\left(\frac{\rho}{2 \rho+a_{0}}\right) t\right|<\left(\frac{\rho+a_{0}}{2 \rho+a_{0}}\right) t .
$$

That proves Theorem 1 . 
Proof of Theorem 2: Since the function $f(z)=\sum_{j=0}^{\infty} a_{j} z^{j}$ is analytic for $|z| \leq t$, it follows that the function

$$
g(z)=f(t z)=\sum_{j=0}^{\infty} a_{j} t^{j} z^{j}
$$

is analytic for $|z| \leq 1$. Consider the function

$$
\begin{aligned}
G(z) & =(z-1) g(z) \\
= & (z-1)\left(a_{0}+a_{1} t z+a_{2} t^{2} z^{2}+\ldots \ldots\right) \\
= & -a_{0}+\left(a_{0}-t a_{1}\right) z+\sum_{j=2}^{\infty}\left(a_{j-1} t^{j-1}-a_{j} t^{j}\right) z^{j} \\
= & -a_{0}+\left(a_{0}-t a_{1}\right) z+z F(z),
\end{aligned}
$$

where

$$
F(z)=\sum_{j=2}^{\infty}\left(a_{j-1} t^{j-1}-a_{j} t^{j}\right) z^{j-1}
$$

Now by hypothesis

$$
\left|\left(\alpha_{1}-t \alpha_{2}\right)+\left(\alpha_{2}-t \alpha_{3}\right) z+\left(\alpha_{3}-t \alpha_{4}\right) z^{2}+\ldots .\right| \leq \frac{M_{1}}{t} \text { for }|z|=t
$$

and

$$
\left|\left(\beta_{1}-t \beta_{2}\right)+\left(\beta_{2}-t \beta_{3}\right) z+\left(\beta_{3}-t \beta_{4}\right) z^{2}+\ldots .\right| \leq \frac{M_{2}}{t} \text { for }|z|=t .
$$

or

$$
t \mid\left(\alpha_{1}-t \alpha_{2}\right)+\left(\alpha_{2}-t \alpha_{3}\right) z+\left(\alpha_{3}-t \alpha_{4}\right) z^{2}+\ldots . . \leq M_{1} \text { for }|z|=t
$$

and

$$
t \mid\left(\beta_{1}-t \beta_{2}\right)+\left(\beta_{2}-t \beta_{3}\right) z+\left(\beta_{3}-t \beta_{4}\right) z^{2}+\ldots . . \leq M_{2} \text { for }|z|=t .
$$

Equivalently

$$
\begin{aligned}
& \mid\left(\alpha_{1}-t \alpha_{2}\right) z+\left(\alpha_{2}-t \alpha_{3}\right) z^{2}+\left(\alpha_{3}-t \alpha_{4}\right) z^{3}+\ldots . . \leq M_{1} \text { for }|z|=t \\
& \text { and } \\
& \left|\left(\beta_{1}-t \beta_{2}\right) z+\left(\beta_{2}-t \beta_{3}\right) z^{2}+\left(\beta_{3}-t \beta_{4}\right) z^{3}+\ldots . .\right| \leq M_{2} \text { for }|z|=t .
\end{aligned}
$$

Replacing $\mathrm{z}$ by tz in the above inequalities, we get

$$
\mid\left(\alpha_{1}-t \alpha_{2}\right) t z+\left(\alpha_{2}-t \alpha_{3}\right) t^{2} z^{2}+\left(\alpha_{3}-t \alpha_{4}\right) t^{3} z^{3}+\ldots . . \leq M_{1} \text { for }|z|=1
$$

and

$$
\left|\left(\beta_{1}-t \beta_{2}\right) t z+\left(\beta_{2}-t \beta_{3}\right) t^{2} z^{2}+\left(\beta_{3}-t \beta_{4}\right) t^{3} z^{3}+\ldots .\right| \leq M_{2} \text { for }|z|=1 \text {. }
$$

Hence, for $|z|=1$,

$$
\begin{aligned}
&|F(z)|=\left|\sum_{j=2}^{\infty}\left(a_{j-1} t^{j-1}-a_{j} t^{j}\right) z^{j-1}\right| \\
&=\mid \sum_{j=2}^{\infty}\left[\left\{\alpha_{j-1} t^{j-1}-\alpha_{j} t^{j}\right\} e^{i \phi} z^{j-1}+\left\{\beta_{j-1} t^{j-1}-\beta_{j} t^{j}\right\} e^{i \psi} z^{j-1}\right] \\
& \leq \mid\left(\alpha_{1}-t \alpha_{2}\right) t z+\left(\alpha_{2}-t \alpha_{3}\right) t^{2} z^{2}+\left(\alpha_{3}-t \alpha_{4}\right) t^{3} z^{3}+\ldots . . \\
& \quad+\left|\left(\beta_{1}-t \beta_{2}\right) t z+\left(\beta_{2}-t \beta_{3}\right) t^{2} z^{2}+\left(\beta_{3}-t \beta_{4}\right) t^{3} z^{3}+\ldots . .\right| \\
& \leq M_{1}+M_{2} .
\end{aligned}
$$


Clearly $\mathrm{F}(\mathrm{z})$ is analytic for $|z| \leq 1$ and $\mathrm{F}(0)=0$. Therefore applying Schwarz lemma to the function $\mathrm{F}(\mathrm{z})$, we get

$$
|F(z)| \leq\left(M_{1}+M_{2}\right)|z| \text { for }|z| \leq 1 \text {. }
$$

Hence for $|z| \leq 1$,

$$
\begin{aligned}
|G(z)| & \geq\left|-a_{0}+\left(a_{0}-t a_{1}\right) z\right|-\left(M_{1}+M_{2}\right)|z|^{2} \\
& \geq\left|a_{0}\right|-\left|a_{0}-t a_{1}\right||z|-\left(M_{1}+M_{2}\right)|z|^{2} \\
& =\left(M_{1}+M_{2}\right)(A-|z|)(|z|+B)
\end{aligned}
$$

where

$$
A=\frac{1}{2\left(M_{1}+M_{2}\right)}\left\{-\left|a_{0}-t a_{1}\right|+\sqrt{\left|a_{0}-t a_{1}\right|^{2}+4\left|a_{0}\right|\left(M_{1}+M_{2}\right)}\right\}
$$

and

$$
B=\frac{1}{2\left(M_{1}+M_{2}\right)}\left\{\left|a_{0}-t a_{1}\right|+\sqrt{\left|a_{0}-t a_{1}\right|^{2}+4\left|a_{0}\right|\left(M_{1}+M_{2}\right)}\right\} .
$$

Clearly $\delta>0$ and for $|z| \leq 1,|G(z)|>0$ if $|z|<\gamma$. Hence it follows that $\mathrm{G}(\mathrm{z})$ and therefore $\mathrm{f}(\mathrm{z})$ does not vanish in $|z|<A t$, which is equivalent to the desired result. This completes the proof of Theorem 2.

\section{REFERENCES}

[1]. A. Aziz and Q. G. Mohammad, On the zeros of a certain class of polynomials and related analytic functions, J. Math. Anal. Appl.75(1980), 495-502.

[2]. A. Aziz and W. M. Shah, On the location of zeros of polynomials and related analytic functions, Nonlinear Studies, Vol.6, No.1, 1999, 91-101.

[3]. V. K. Jain, On the zeros of a class of polynomials and related analytic functions, J.Pure Appl. Math. 28(1997), 533-549.

[4]. C. Karanicoloff, A theorem of Enestrom-Kakeya type, Math.Lopok 14 (1963), 133136(Hungarian, Russian and English Summaries).

[5]. M. Marden, Geometry of Polynomials, IInd . Ed. Math. Surveys 3, Amer. Math. Soc., Providence, RI(1966). 